\title{
Antibody response against cancer-testis antigens MAGEA4 and MAGEA10 in patients with melanoma
}

\author{
KADRI ÕUNAP $^{1 *}$, KRISTIINA KURG $^{1 *}$, LIISI VÕSA $^{1}$, ÜLO MAIVÄLI $^{1}$, \\ MARINA TERAS ${ }^{2}$, ANU PLANKEN $^{2}$, MART USTAV $^{1}$ and REET KURG ${ }^{1}$ \\ ${ }^{1}$ Institute of Technology, University of Tartu, 50411 Tartu; ${ }^{2}$ Melanoma Unit of The General Surgery and \\ Oncology Surgery Centre, North Estonian Medical Centre, 13419 Tallinn, Estonia
}

Received October 20, 2017; Accepted April 19, 2018

DOI: $10.3892 / 01.2018 .8684$

\begin{abstract}
Melanoma-associated antigen A (MAGEA) represent a class of tumor antigens that are expressed in a variety of malignant tumors, however, their expression in healthy normal tissues is restricted to germ cells of testis, fetal ovary and placenta. The restricted expression and immunogenicity of these antigens make them ideal targets for immunotherapy in human cancer. In the present study the presence of naturally occurring antibodies against two MAGEA subfamily proteins, MAGEA4 and MAGEA10, was analyzed in patients with melanoma at different stages of disease. Results indicated that the anti-MAGEA4/MAGEA10 immune response in melanoma patients was heterogeneous, with only $\sim 8 \%$ of patients having a strong response. Comparing the number of strongly responding patients between different stages of disease revealed that the highest number of strong responses was detected among stage II melanoma patients. These findings support the model that the immune system is involved in the control of melanoma in the early stages of disease.
\end{abstract}

\section{Introduction}

Melanoma-associated antigen A (MAGEA) subfamily proteins are members of cancer/testis antigens (CTAs), whose normal expression is limited to germ cells, but ectopic expression can be observed in tumor cells of different origins (1). The MAGEA genes were initially identified as tumor antigens that can be recognized by cytotoxic T-lymphocytes in melanoma patients (2). The MAGEA subgroup of CTA family comprises eleven genes that show striking homology with each other and are encoded as a cluster at the Xq28 region (3).

Correspondence to: Dr Reet Kurg, Institute of Technology, University of Tartu, Nooruse Street 1, 50411 Tartu, Estonia

E-mail: reet.kurg@ut.ee

*Contributed equally

Key words: cancer-testis antigens, melanoma antigens, melanoma, antibodies, cancer
Their normal expression is restricted to the testis, trophoblast and placenta $(3,4)$. MAGEA expression in somatic cells is silenced by promoter DNA methylation (5), but in tumor cells genome-wide epigenetic reprogramming can result in promoter hypo-methylation, leading to aberrant expression of one or more of these genes $(1,6)$.

MAGEA expression is observed mainly in cancers that have acquired malignant phenotypes, invasiveness or metastasis, and the expression of MAGEA family proteins has been linked to poor prognosis in cancer patients. MAGEA family proteins have oncogenic functions, including support of growth, survival and metastasis, and are thought to contribute actively to malignancy (7). At the molecular level, MAGEA proteins are involved, through direct and indirect mechanisms, in the regulation of the tumor suppressor protein $\mathrm{p} 53$ pathway (8-12). MAGEA proteins can also activate specific RING finger type E3 ubiquitin ligases $(13,14)$, thereby regulating the ubiquitin signaling in cancer cells.

MAGEA proteins are known to be highly expressed in a wide range on cancers including bladder, lung, skin and breast malignancies $(6,15-18)$. Expression of these antigens may be highly heterogeneous in a variety of tumors of different histological origin, with percentages of positive cells ranging between 5 and $60 \%$ (18). MAGEA subfamily proteins are highly conserved and it is very difficult to get antibodies that recognize only one member of the family specifically. For example, MAGEA4 and MAGEA10 proteins share more than $50 \%$ sequence identity on the amino acid level, but have different sizes and cellular localizations (19). Several antibodies used in immunohistochemical studies cross-react with many MAGEA proteins and have been seen in multiple cancer types to localize both in the cytoplasm and in the nucleus (20-22). This has complicated the immunohistochemical analysis of cancer tissues and limited the analysis of specific subfamily members, which may have different expression patterns, subcellular localizations and impacts on the malignancy.

Melanoma is the most serious type of skin cancer and its incidence has risen over the years. The etiology of melanoma is multi-factorial, resulting from gene-environment interactions, with the main environmental factor for melanoma development being exposure to sunlight and UV radiation (23). The importance of the immune system in the etiology of human skin cancer has been long recognized, based primarily upon 
the increased incidence of skin cancers in organ transplant recipients and mechanisms of ultraviolet (UV) radiation-mediated immunomodulation (24). Although the rate of melanoma incidence is rising, especially within young females, there is no direct correlation with the increase of mortality. Histological regression in primary cutaneous melanoma has been shown to occur in $10-35 \%$ of cases (25). Thus, it can be hypothesized that the immune system is involved in controlling the melanoma progression, especially in younger individuals.

The aim of this study was to evaluate the presence of naturally occurring antibodies against two MAGEA proteins in the blood samples of melanoma patients with different stages of disease. MAGEA proteins have oncogenic functions contributing to malignancy, and they are known to be immunogenic proteins. The MAGEA4 and MAGEA10 proteins were expressed in bacteria, purified and used in the enzyme-linked immunosorbent assay (ELISA) for detection of antibodies. We were curious to know i) whether the melanoma patients have antibodies against these proteins, and ii) whether these antibodies can be treated as a potential prognostic marker.

\section{Materials and methods}

Patients and sera. Human sera were obtained from 185 patients with melanoma attending the North Estonian Medical Centre (Tallinn, Estonia) within two years (2013-2014). The melanoma stage was assigned based on tumor thickness, ulceration and the involvement of lymph nodes or organs. The characteristics of patients are shown in Table I. As a control, we included 43 sera of healthy blood donors from the Estonian Blood Bank. We have no data about the gender nor age of blood bank controls. All samples were handled by standard procedures and stored at $-80^{\circ} \mathrm{C}$. Approval for the use of blood samples for the study was obtained from the Tallinn Medical Research Ethics Committee (Tallinn, Estonia).

Proteins. MAGEA4 and MAGEA10 coding sequences from pQMCF-MAGEA4 and pQMCF-MAGEA10 vectors (19) were cloned in frame into pET28a vector using NheI restriction enzyme to fuse the coding sequence with His-tag. Recombinant His-tagged MAGEA4 and MAGEA10 proteins were transformed into Escherichia coli $\left(E\right.$ coli) cells BL-CodonPlus ${ }^{\mathrm{TM}} \mathrm{RP}$ (Invitrogen; USA); transformed bacteria were grown at $37^{\circ} \mathrm{C}$ to the spectrophotometric density 0.6 (OD $600 \mathrm{~nm}$; Ultraspec 7000; GE Healthcare Life Sciences, Little Chalfont, UK) and induced with $1 \mathrm{mM}$ IPTG for $2 \mathrm{~h}$ at room temperature. Then the cells were collected by centrifugation (at 8,000 x $\mathrm{g}$ for $3 \mathrm{~min}$ at $4^{\circ} \mathrm{C}$; Centrifuge 5810R; Eppendorf, Hamburg, Germany) and resuspended in buffer containing $50 \mathrm{mM}$ Tris $(\mathrm{pH} 8.0)$ and $500 \mathrm{mM} \mathrm{NaCl}$. Proteins were purified with Ni-Sepharose ${ }^{\mathrm{TM}} 6$ Fast Flow beads (GE Healthcare Life Sciences) under standard native conditions following manufacturer's recommendations; $20 \mathrm{mM}$ imidazole was added to the buffer for binding reactions, $25 \mathrm{mM}$ for wash buffers and $250 \mathrm{mM}$ for elution of proteins from the beads. Both proteins were purified using the same protocol. After purification, the buffer was exchanged to PBS with Amicon ${ }^{\circledR}$ Ultra centrifugal filters (Sigma-Aldrich; Merck KGaA, Darmstadt, Germany) and the concentration of proteins was determined by the Bradford assay using bovine serum albumin (BSA) as a standard.
ELISA. Recombinant MAGEA4 orMAGEA10 protein $(2 \mu \mathrm{g} / \mathrm{ml})$ in phosphate-buffered saline (PBS) containing $0.1 \%$ of Tween-20 was adsorbed onto 96-well MaxiSorp NUNC-immunoplates (Sigma-Aldrich; Merck KGaA) and incubated overnight at $4^{\circ} \mathrm{C}$. Plates were washed with PBS/0.1\% Tween-20 and blocked with $2 \%$ BSA in PBS $/ 0.1 \%$ Tween-20. Serial dilutions of human serum in $100 \mu \mathrm{l}$ of $0.4 \% \mathrm{BSA} / \mathrm{PBS} / 0.1 \%$ Tween-20 were added to each well and incubated for one hour at room temperature on the shaker (Titertek-Berthold; Berthold Detection Systems $\mathrm{GmbH}$, Pforzheim, Germany). Horseradish peroxidase (HRP)-conjugated goat anti-human IgG (Zymax/Invitrogen; Thermo Fisher Scientific, Inc., Waltham, MA, USA) was used as a secondary antibody for $45 \mathrm{~min}$. After washing four times, the reaction was developed with the TMB Peroxidase E1A substrate kit (Bio-Rad Laboratories, Inc., Hercules, CA, USA) for $10 \mathrm{~min}$. and stopped with $\mathrm{H}_{2} \mathrm{SO}_{4}$. The absorbance at $450 \mathrm{~nm}$ was measured spectrophotometrically using the ELISA plate reader Sunrise ${ }^{\mathrm{TM}}$ (Tecan, Männedorf, Switzerland). For quality control, we included three reference sera which were analyzed on every ELISA plate. The CVs of their ODs did not exceed $20 \%$.

Statistical analysis. The data were analyzed in R (version 3.3.0). Parameter estimates and corresponding CI (credible intervals) were calculated using the BayesFirstAid package (26). Analysis of variance (ANOVA) with the Tukey post-test was also done in $\mathrm{R}$.

The patients with positive antibody response were defined as follows: pooled MAGEA4 and MAGEA10 response values obtained from the blood bank donors were log-transformed to ensure normality, after which the mean and the standard deviation was calculated from the control subjects only. Then the melanoma patients, whose log-response value $>$ mean + $2^{*} \mathrm{SD}$, were redefined as having a strong response. To classify subjects based on their MAGEA protein levels a logistic regression model, including both MAGEA proteins, sex, and age as additive predictors, was trained on the subset of data containing stage 0 , I, and II patients. The pROC package was used to calculate the receiver operating characteristic (ROC) curve (27).

\section{Results}

Antibody response against MAGEA4 and MAGEA10 proteins in melanoma patients. We measured the anti-MAGEA4 and anti-MAGEA10 antibody levels by ELISA from 185 stage 0 (in situ) to stage IV melanoma patients and from 43 healthy individuals, who had donated their blood to the Estonian blood bank. The ELISA was performed using MAGEA4 and MAGEA10 proteins, which were purified from E. coli, immobilized on microtiter plates, and subsequently probed with 1:200 to 1:800 human sera dilutions. The serums that exhibited high OD values, indicating the presence of anti-MAGEA antibodies, were tested at least three times on separate ELISA plates and the mean OD value was used in further analysis. The OD values obtained from 1:400 diluted serums were used in statistical analysis.

We first compared the OD values of the controls with the melanoma patients separately for anti-MAGEA4 and anti-MAGEA10 response (Fig. 1). In Fig. 1, the Tukey box 
Table I. Characteristics of the melanoma patients.

\begin{tabular}{|c|c|c|c|c|c|c|}
\hline Number & $\begin{array}{c}\text { Stage } 0 \\
24\end{array}$ & $\begin{array}{c}\text { Stage I } \\
67\end{array}$ & $\begin{array}{c}\text { Stage II } \\
43\end{array}$ & $\begin{array}{l}\text { Stage III } \\
30\end{array}$ & $\begin{array}{c}\text { Stage IV } \\
21\end{array}$ & $\begin{array}{c}\text { Total } \\
185\end{array}$ \\
\hline \multicolumn{7}{|l|}{ Sex } \\
\hline Male $(\%)$ & $4(16.7 \%)$ & $17(25.4 \%)$ & $14(32.6 \%)$ & $12(40 \%)$ & $9(42.9 \%)$ & $56(30.3 \%)$ \\
\hline Female (\%) & $20(83.3 \%)$ & $50(74.6 \%)$ & $29(67.4 \%)$ & $18(60 \%)$ & $12(57.1 \%)$ & $129(69.7 \%)$ \\
\hline \multicolumn{7}{|l|}{ Disease duration } \\
\hline$<5$ years & $21(87.5 \%)$ & $47(70.1 \%)$ & $29(67.4 \%)$ & $20(66.7 \%)$ & $18(85.7 \%)$ & $135(73 \%)$ \\
\hline$\geq 5$ years & $3(12.5 \%)$ & $20(29.9 \%)$ & $14(32.6 \%)$ & $10(33.3 \%)$ & $3(14.3 \%)$ & $50(27 \%)$ \\
\hline Mean (range) & $2.0(0-13)$ & $4.5(0-26)$ & $3.7(0-18)$ & $4.5(0-19)$ & $2.8(0-25)$ & $3.8(0-26)$ \\
\hline Median & 1 & 2 & 3 & 3 & 1 & 2 \\
\hline \multicolumn{7}{|l|}{ Age } \\
\hline Mean (range) & $51.9(18-87)$ & $61.3(28-87)$ & $64.3(33-90)$ & $63.4(43-82)$ & $73.1(35-92)$ & $62.5(18-92)$ \\
\hline Median & 51 & 65 & 66 & 65 & 78 & 65 \\
\hline
\end{tabular}

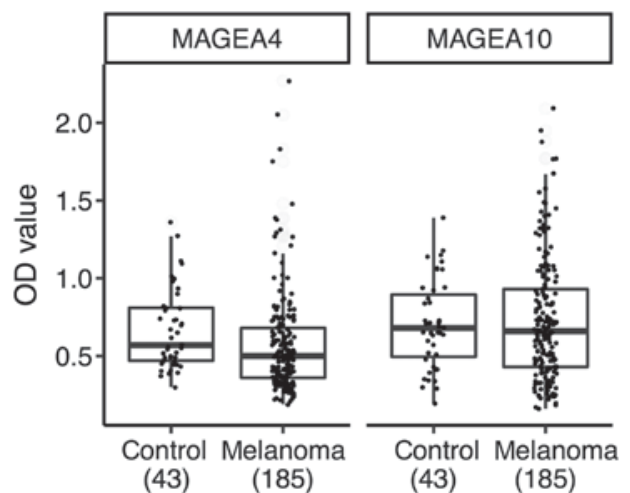

Figure 1. Antibody response against MAGEA4 and MAGEA10 proteins in melanoma patients and controls. A comparison of the magnitude of the anti-MAGEA4 and anti-MAGEA10 immune responses of blood bank controls vs. melanoma patients (stages 0-IV combined) by Tukey box plots showing median and interquartile ranges. Dots correspond to individual blood bank controls and patients. The Y-axis denotes optical density values obtained from the ELISA assay. The number of sera is shown in the parentheses. MAGEA, melanoma-associated antigen A.

blots were used to show the median and interquartile ranges, as well as dots corresponding to individual patients and blood bank controls. The mean immune responses of patients were not elevated as compared with the blood bank controls. The mean OD value of the sera of melanoma patients was 0.59 (SD 0.31) for MAGEA4 and $0.73(0.38)$ for MAGEA10. For blood bank controls, the mean OD value was $0.67(0.26)$ for MAGEA4 and $0.70(0.28)$ for MAGEA10. We could not find evidence for elevated mean effects of melanoma patients over blood bank controls, the probability that the patients mean value was higher than the controls was $0.3 \%$ for MAGEA4 (two-sided P-value $=0.004)$ and $62 \%$ for MAGEA10 $(\mathrm{P}=0.87)$. On the other hand, the patients had higher variability at their anti-MAGEA4 and anti-MAGEA10 responses than controls; the probability that the controls have higher standard deviations was 2 and $6 \%$ for MAGEA4 and MAGEA10, respectively. We suggest that the higher variability of the immune response in patients could mean that in some patients the antibody response is induced, while in others is not. The failure of the patients to exhibit aggregate effects over the controls is likely due to the voluntary blood donors, who make up the control group. We have no data about their age and gender, but they are probably younger than the melanoma patients. This limits the usefulness of the blood donors as controls. Therefore, in the subsequent analysis we omitted the blood bank controls and looked at melanoma stages 0 to IV as distinct groups.

To follow the antibody response from limited to advanced disease, we divided the patients into subgroups, depending on the status of their disease. In Fig. 2, the Tukey box blots were used to show the median and interquartile ranges, as well as dots corresponding to individual patients; the number of patients is shown in parenthesis under the stage number. As shown in Fig. 2, some stage I, II and III patients exhibited elevated anti-MAGEA4 and/or anti-MAGEA10 immune responses. The one-way ANOVA P-value was 0.10 for MAGEA4 and 0.043 for MAGEA10, indicating that there are statistically significant contrast(s) in the MAGEA10 data. We used the Tukey HSD post-test to find groups that significantly differ from each other. This showed that in the case of MAGEA10, there was a single contrast, stage 0 vs. stage II patients, which had a significant difference in mean $\mathrm{OD}$ values $(\mathrm{P}=0.047)$. The mean $\mathrm{OD}$ values between stage II and stage IV patients were slightly different $(\mathrm{P}=0.10)$, but no difference was observed between stage II and III patients $(\mathrm{P}=0.78)$. In the case of MAGEA4, we did not observe statistically significant differences in mean values between patients with different stages of disease $(\mathrm{P}=0.74$ for stage 0 vs. stage II; $\mathrm{P}=0.18$ for stage II vs. stage IV and $\mathrm{P}=0.47$ for stage II vs. stage III). These data show that there is no strong difference in mean OD values between stage II and III, but is a slight difference between stage II and IV for MAGEA10. Although, we found only a single statistically significant contrast, due to the limited sample size this does not necessarily mean that there are no real differences between stage II and III\&IV. We sought to clarify this point further by polynomial regression modelling. We predicted anti-MAGEA4/A10 response levels (as measured by OD) from the stage of melanoma modelled as a continuous ordinal variable. These models indicate that for both proteins there 
Table II. Patients with strong antibody response.

\begin{tabular}{llcrll}
\hline Patient & Gender & Age (years) & Disease duration (years) & Stage & Protein \\
\hline M35 & F & 61 & 2 & IIB & MAGEA4 value \\
M38 & F & 73 & 18 & II & MAGEA4 \\
M111 & M & 67 & 1 & IIIC & MAGEA4 \\
M123 & F & 65 & 0 & IB & MAGEA4 \\
M162 & M & 64 & 3 & IA & MAGEA4 \\
M3 & F & 57 & 1 & IB & MAGEA10 \\
M38 & F & 73 & 18 & II & MAGEA10 \\
M47 & F & 71 & 4 & IIB & MAGEA10 \\
M63 & F & 80 & 3 & IIIA & MAGEA10 \\
M70 & F & 64 & 9 & IIB & MAGEA10 \\
M76 & F & 61 & 6 & IIB & MAGEA10 \\
M99 & F & 66 & 5 & IIB & MAGEA10 \\
M11 & M & 67 & 1 & IIIC & MAGEA10 \\
M115 & M & 72 & 1 & IIB & MAGEA10 \\
M119 & F & 76 & 11 & IB & MAGEA10 \\
M137 & F & 72 & 0 & IIB & MAGEA10 \\
M144 & F & 52 & 1 & IIIA & MAGEA10 \\
\hline
\end{tabular}

${ }^{a, b}$ These patients have a strong antibody response against MAGEA4 and MAGEA10 protein. MAGEA, melanoma-associated antigen A.
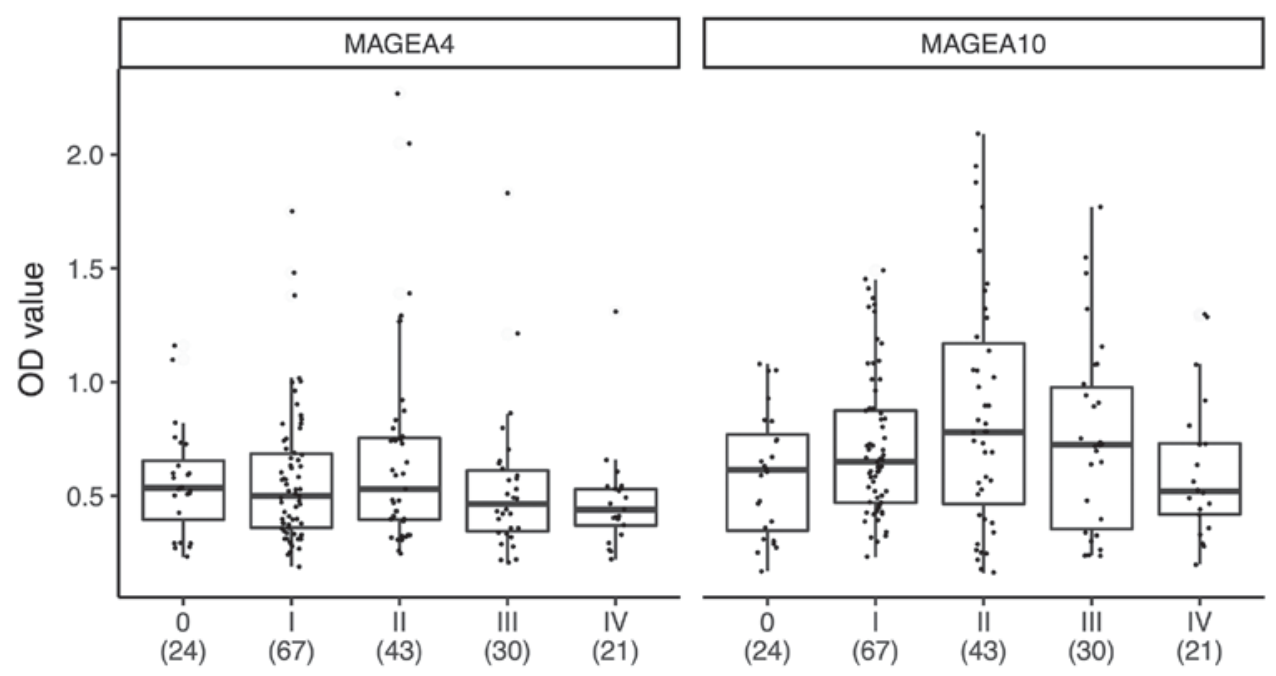

Figure 2. A subgroup analysis of the magnitude of the anti-MAGEA4 and anti-MAGEA10 immune responses of melanoma patients (stages 0-IV). Tukey box plots with median and interquartile ranges are shown, as well as dots corresponding to individual blood bank controls and patients. The Y-axis denotes optical density values at $495 \mathrm{~nm}$. The number of sera is shown in the parenthesis. MAGEA, melanoma-associated antigen A.

is an initial rise in optical density that peaks at stage II, and thereafter falls again (data not shown).

As our samples were not balanced for age and sex (Table I), we also looked for associations between these variables and anti-MAGE response. By applying linear regression towards our stage 0 to IV melanoma samples, we could find no significant association between the age of the subjects and anti-MAGEA4 or anti-MAGEA10 response (data not shown). However, we found a weak association between anti-MAGEA10 levels and sex $\left(r^{2}=0.025\right.$; female melanoma patients have on average 0.15 OD units higher anti-MAGEA10 response than male patients, 95\% CI: 0.04, 0.26). But the width of the CI indicates that our sample size is not large enough to decide whether this effect is scientifically relevant.

Patients with strong antibody response. Next, we focused on patients with strong anti-MAGEA4 and/or anti-MAGEA10 immune responses. Here we included patients whose OD values were higher than the mean OD of the healthy blood bank donors plus 2 SD-s (28). Table II shows the patients with strong antibody response. The sera of 15 patients from 185 (8.2; $95 \%$ CI: $4.7,13 \%$ ) had a strong antibody response against the MAGEA4 and/or MAGEA10 protein (Table II). Two patients (M38 and M111) had a strong response against both proteins, 


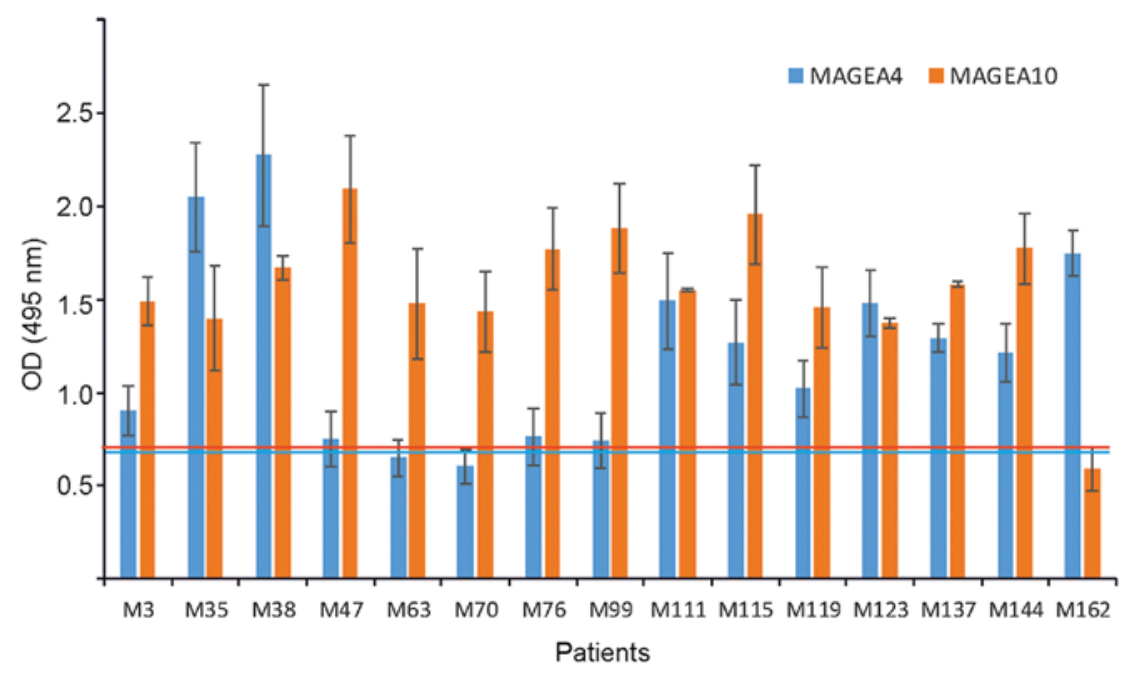

Figure 3. Comparison of OD values of MAGEA4 and MAGEA10 among the strongly responding patients. Lines correspond to mean values of ELISA assay for MAGEA4 (blue) and MAGEA10 (orange), respectively. Error bars show the SD of at least three different experiments performed on separate ELISA plates. MAGEA, melanoma-associated antigen A.

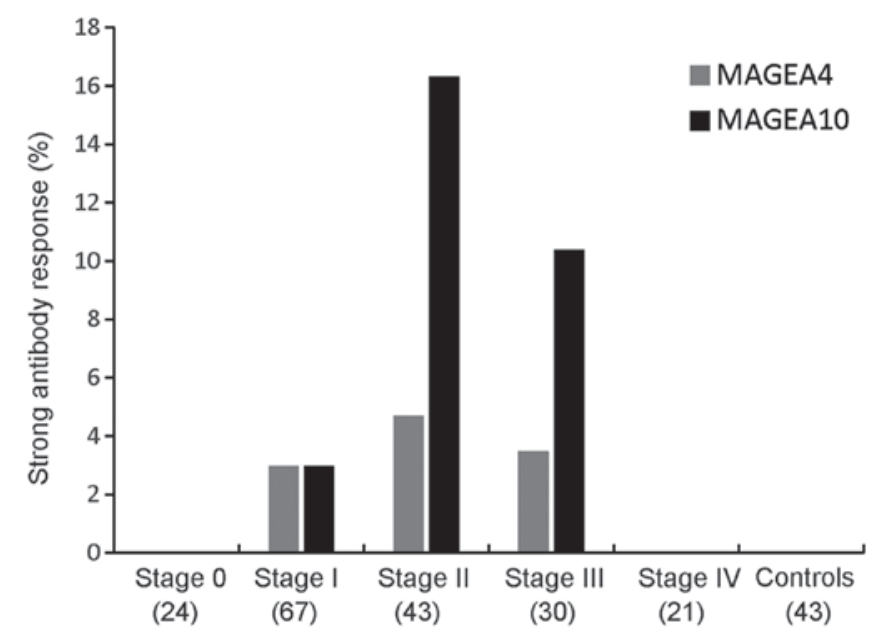

Figure 4. The fraction of strongly responding patients in relation to the melanoma stage. Patients with OD values higher than the mean OD of the healthy blood bank donors plus 2 SD-s are included. The number of sera is shown in the parenthesis. MAGEA, melanoma-associated antigen A.

that is why there are 17 patients listed in Table II. The mean age of strongly responding patients was 67 years (median 67 years) and they were first diagnosed from 0 to 18 years (mean 5 years, median 3 years) before this analysis was performed. Most of them are women, there are only 3 men (20; 95\% CI: $5.4,43 \%)$ among the patients with strong antibody response, while the whole cohort consists of $30.3 \%$ of men. Altogether, $5.6 \%$ of men and $9.3 \%$ of women had strong antibody response against one or two of the MAGEA proteins.

MAGEA proteins are highly similar to each other with half of the amino acids identical between MAGEA4 and MAGEA10 proteins. We have analyzed the sera separately for MAGEA4 and MAGEA10 response, and the statistics was performed and cut-off values calculated independently of each other. Interestingly, there were 12 anti-MAGEA10 responses and 5 anti-MAGEA4 responses, out of the total of 17 strong responses (estimated relative frequency of MAGEA4 is 0.31 ; $95 \%$ CI: $0.13,0.52 ; 5 \%$ probability of relative frequency $>0.5$ )

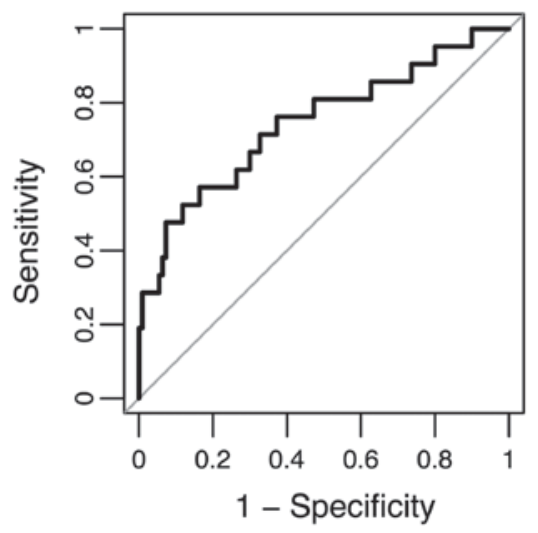

Figure 5. ROC curve for anti-MAGE antibody detection. Antibody levels among 185 melanoma patients and 43 blood bank controls were determined by ELISA. The AUC value was 0.74 . ROC, receiver operating characteristic; MAGE, melanoma-associated antigen; AUC, area under the curve.

(Table II). Consequently, only two patients out of 15 (13.3, 95\% CI: $2.7,35 \%$ ) had strong and statistically significant responses against both, MAGEA4 and MAGEA10 proteins. In Fig. 3, we compare the antibody response against two different antigens among the same patients. Some patients (M38, M111, but also M35, M123 and M137) had antibodies against both MAGE-A proteins, the others (M47, M63, M70, M76, M99 and M162) against only one of the two proteins, either MAGEA4 or MAGEA10. Five patients of $15(33 \%)$ had a statistically significant higher $\mathrm{OD}$ value $(\mathrm{P}<0.01)$ against MAGEA10 than MAGEA4, while only one patient, M162, had a better immune response against MAGEA4 (Fig. 3). These data show that among strongly responding patients, there are more anti-MAGEA10 than anti-MAGE4 responses.

Comparing the number of strongly responding patients between different stages of disease revealed that the highest number of strong responses was detected among stage II melanoma patients (Fig. 4). In the case of MAGEA10, 7 of 43 (16.3, 95\% CI: 7.3, 29\%) sera were positive among stage II patients, and 3 of $29(10.3,95 \%$ CI: $2.4,24 \%)$ in stage III patients. In 
the case of MAGEA4, there was no clear preference to any stage, strongly responding sera belonged to patients of stages I, II and III. We could not detect any strong response from the blood samples of melanoma patients with stage 0 and IV.

Predictive modelling of anti-MAGE-A responses. To explore the potential diagnostic value of anti-MAGEA antibodies, we classified all stage 0 vs. pooled stage I and II patients using an additive logistic regression model that includes both MAGEA proteins, age, and sex. We summarized the model performance in a ROC curve where we plotted the sensitivity (true positive rate) values against 1 -specificity (false positive rate) values for each possible cut-point (Fig. 5). The area under the curve (AUC) is 0.74 , suggesting that anti-MAGEA antibodies can be treated as potential diagnostic biomarkers.

\section{Discussion}

MAGEA proteins are cancer-testis antigens (CTAs), which elicit both cellular and humoral responses. In this study, we have analyzed the presence of naturally occurring antibodies against two MAGEA family proteins, MAGEA4 and MAGEA10, in melanoma patients with different stages of disease. Our data showed that sera of 15 patients out of 185 (8\%) had a strong antibody response against the MAGEA4 and/or MAGEA10 protein. The highest antibody response was detected in stage II melanoma patients.

CTAs are named after their typical pattern of expression in a variety of malignant tumors. Their expression in normal tissues is restricted to germ cells of the testis. Male germ cells are devoid of HLA-class I molecules and cannot present antigens to T cells. Therefore, MAGEA antigens can be considered neo-antigens when expressed in cancer cells (29). Recent studies have shown that the induction of MAGEA4-specific immune responses correlated well with the prognosis of patients vaccinated with MAGEA4 protein and that antibody response could be a marker for a good prognosis (28).

Our study revealed that $8 \%$ of patients had strong antibody responses against the MAGEA4 or/and MAGEA10 protein. When we grouped patients according to the level of the disease, then stage II patients had more antibodies than others, reaching to $16 \%$ in case of MAGEA10. Scultz-Thater et al have studied the prevalence of MAGEA10 in different cancers and shown that it is expressed in $38 \%$ of malignant melanomas (18). Several studies have shown that MAGEA proteins are associated with or contribute to solid malignancies, MAGE-A expression is considered to be an important predictor of malignant transformation (21). For instance, MAGEA4 is expressed in $9 \%$ of primary tumors, but reaching to $44 \%$ in distant metastasis (30) and MAGEA1 expression has been found in $16 \%$ of primary melanomas and $48 \%$ of metastatic melanomas (15). However, in another study no correlation was observed, and MAGEA3/4 protein was present in $25 \%$ of primary invasive and metastatic tumors, but not in in situ melanomas (31). In our study, the prevalence of MAGEA antibodies was highest in stage II and lowest in stage 0 and stage IV patients. We have not determined the expression of MAGEA proteins in the tissue samples of our patients, but it is very unlikely that MAGEA expression declines in advanced stages. Previous studies have shown that MAGEA4 is rarely lost when once acquired (30). We favor the explanation that stage II melanoma patients have a better immune response than patients with more advanced stages of disease. This is consistent with the immune evasion seen in metastatic cancers (32). Interestingly, there were also very few responses amongst in situ and stage I melanoma patients. This can be explained by the localization of the primary tumor. Stage 0 or in situ and stage I melanoma are found mostly on the outer layer of the skin, in epidermis. Stage II melanoma has spread to the lower part of the inner layer of skin (dermis), but not yet into the tissue below the dermis or into nearby lymph nodes. The dermis contains many antigen presenting cells, which may help to boost the immune response.

In our study, some patients had strong antibody response against both MAGE-A proteins, the others exhibited antibodies against either MAGEA4 or MAGEA10 protein. One of the limitations of this study is that we do not have biopsies of patients and we were not able to perform neither qPCR nor immunochemical analysis to confirm that the antibodies are specific to MAGEA4 or MAGEA10. However, tumor cells very often express more than one MAGEA protein. Simultaneous expression of five or more MAGEA proteins occurs in more than half of oral squamous cell carcinomas (33) and simultaneous expression of MAGEA1 and MAGEA4 expression occurs in $60-70 \%$ of melanomas (30). We favor the explanation that the two MAGEA proteins used in our study have different immunogenic properties, so that the MAGEA10 protein is a better antigen than MAGEA4. Thus, our work is consistent with the studies, which have suggested that MAGEA10 is the most immunogenic antigen of the MAGEA family (34-36). It is well known that obtaining antibodies against one specific MAGEA protein may be challenging; we cannot rule out the possibility that antibodies detected in our assay are formed against some other member of the family. MAGEA proteins are highly similar to each other, with half of the amino acids identical between MAGEA4 and MAGEA10. The MAGEA subfamily consists of 11 MAGE-A proteins and in addition, there are MAGE-B, MAGE-C, MAGE-D etc. families which all share the MHG (MAGE homology) domain (37). All these proteins are to some extent similar to each other (MHG domains has similarities from 25 to 80\%) and may give some cross-reactivity. This may also explain the immune response against MAGEA proteins in some healthy donors seen in our study. The other limitation of our study is that we have used only ELISA assay for screening of the sera. We choose ELISA, because it is suitable for high-throughput analysis, but have not analyzed the sera by western blotting. However, by doing so we might have missed some antibody responses generated against linear and/or denatured epitopes of MAGEA proteins.

The existence of strongly responding patients suggests that their immune system has been activated and has started to generate antibodies against the primary tumor. So, our data support the hypothesis that the immune system is involved in the control of melanoma, at least in the early stages. Several studies have shown spontaneous regression of primary melanomas, but regression of metastatic tumors is very rare. A good antibody response at early stages can stop the growth of primary tumor and further spreading to the lymph nodes and other organs. Among the 15 patients of our study with the positive status for MAGEA antibodies, only one has died 
and one has disease progression during the 2-year post-study follow-up period (data not shown). So, the disease of the majority of patients with strong antibody response is under control. However, this cohort is too small to make long-term conclusions about the prognosis. Longitudinal time-course studies on larger cohorts are needed to establish the prognostic significance of the presence of MAGEA antibodies in patients. We plan to follow the patients and their antibody response for at least five more years and perform then the survival analysis.

The sensitivity and specificity calculations suggest that the anti-MAGEA antibody response can be treated as a potential diagnostic biomarker. One of the limitations for use in clinics is that MAGE-A proteins are expressed only in a portion of cancer cells; different works have shown that the amount of expressing cells is between 25 and 50\% (30-31). When we assume that only half of these people have a strong immune response, then the expected \% of strongly responding patients will be 12 to $25 \%$. Is this enough for clinical diagnostics? On the other hand, these antibodies are so-called early markers and there is a great need for early cancer markers. So, when the presence of strong antibody response correlates with good prognosis, then they are/will be useful for clinics.

In addition, from the clinical aspect, the longitudinal detection of MAGEA antibody levels could be utilized for profiling of disease status or of effectiveness of novel immunotherapies, as there exists a great need for biomarkers which could assist in discrimination of patients suitable for immunotherapy or for monitoring the therapy effectiveness of these expensive drugs. For example, it has been shown that during immunotherapy with ipilimumab the MAGEA protein levels declined and elevation correlated with either treatment response or failure, respectively (38). However, the anti-MAGEA antibody status of patients prior to and after checkpoint therapy has never been evaluated.

In summary, our study supports the role of the host immune response in the progression of melanoma. To the best of our knowledge, the present study is the first report on following the antibody response against MAGEA-s and comparing it with the disease progression. A healthy immune system enables to create antibodies against cancer antigens that are expressed specifically by tumor cells. The link between MAGEA antigens and cancer is widely known and accepted; several works have shown a good cellular and humoral response against MAGEAs $(1,6,37)$. Due to their relatively high tumor specificity, they represent attractive targets for active specific and adoptive cancer immunotherapies (39). In the current study, we are not interested in antigens, but we focus on the antibody response against the antigens. There are some studies who have analyzed the antibodies against melanoma antigens (tyrosinase, and TRPs) in melanoma patients, but not against MAGE-A proteins $(40,41)$. In the current study, we focused on naturally occurring antibody response against MAGEA proteins. We compared the number of strongly responding patients between different stages of disease and found that the highest number of strong responses was detected among stage II melanoma patients. The strong antibody response could be a marker for a good prognosis (28) as well as an early marker which can be used for cancer diagnostics from liquid biopsy.

\section{Acknowledgements}

Not applicable.

\section{Funding}

This study was supported by institutional research funding (IUT20-27) of the Estonian Ministry of Education and Research and by the European Regional Development Fund through the Center of Excellence in Molecular Cell Engineering, and by Estonian Health Program TerVe project IMGEMEL.

\section{Availability of data and materials}

The datasets used and/or analyzed during the current study are available from the corresponding author on reasonable request.

\section{Authors' contributions}

KÕ, KK and RK designed the experiments; KÕ, KK and LV conducted the experiments; MT and AP collected the clinical data and were responsible for ethics approvals and consent of patients to participate in the study; ÜM performed the statistical analysis; MU was responsible for overall the design and funding of the project and KÕ, ÜM, AP and RK prepared the figures and wrote the manuscript. All authors read and have approved the final manuscript.

\section{Ethics approval and consent to participate}

Approval no. 2781 (from June 21, 2012) for the use of blood samples of melanoma patients, and no. 254 (from December 13, 2012) for controls were obtained from the Tallinn Medical Research Ethics Committee of Estonian National Institute for Health Development. All the patients, whose blood samples have been used, had signed the consent to participate in the study.

\section{Consent for publication}

The patients have provided written informed consent that the results of the study are published.

\section{Competing interests}

The authors declare that they have no competing interests.

\section{References}

1. Meek DW and Marcar L: MAGE-A antigens as targets in tumour therapy. Cancer Lett 324: 126-132, 2012.

2. van der Bruggen $P$, Traversari C, Chomez $P$, Lurquin $C$, De Plaen E, Van den Eynde B, Knuth A and Boon T: A gene encoding an antigen recognized by cytolytic $\mathrm{T}$ lymphocytes on a human melanoma. Science 254: 1643-1647, 1991.

3. Chomez P, De Backer O, Bertrand M, De Plaen E, Boon T and Lucas S: An overview of the MAGE gene family with the identification of all human members of the family. Cancer Res 61: 5544-5551, 2001. 
4. Kalejs $\mathrm{M}$ and Erenpreisa $\mathrm{J}$ : Cancer/testis antigens and gametogenesis: A review and 'brain-storming' session. Cancer Cell Int 5: 4, 2005.

5. De Smet C, Lurquin C, Lethé B, Martelange V and Boon T: DNA methylation is the primary silencing mechanism for a set of germ line- and tumor-specific genes with a $\mathrm{CpG}$-rich promoter. Mol Cell Biol 19: 7327-7335, 1999.

6. Sang M, Lian Y, Zhou X and Shan B: MAGE-A family: Attractive targets for cancer immunotherapy. Vaccine 29: 8496-8500, 2011.

7. Liu W, Cheng S, Asa SL and Ezzat S: The melanoma-associated antigen A3 mediates fibronectin-controlled cancer progression and metastasis. Cancer Res 68: 8104-8112, 2008.

8. Marcar L, Ihrig B, Hourihan J, Bray SE, Quinlan PR, Jordan LB, Thompson AM, Hupp TR and Meek DW: MAGE-A cancer/testis antigens inhibit MDM2 ubiquitylation function and promote increased levels of MDM4. PLoS One 10: e0127713, 2015

9. Marcar L, Maclaine NJ, Hupp TR and Meek DW: Mage-A cancer/testis antigens inhibit p53 function by blocking its interaction with chromatin. Cancer Res 70: 10362-10370, 2010.

10. Yang B, O'Herrin SM, Wu J, Reagan-Shaw S, Ma Y, Bhat KM, Gravekamp C, Setaluri V, Peters N, Hoffmann FM, et al: MAGE-A, mMage-b, and MAGE-C proteins form complexes with KAP1 and suppress p53-dependent apoptosis in MAGE-positive cell lines. Cancer Res 67: 9954-9962, 2007.

11. Monte M, Simonatto M, Peche LY, Bublik DR, Gobessi S, Pierotti MA, Rodolfo M and Schneider C: MAGE-A tumor antigens target p53 transactivation function through histone deacetylase recruitment and confer resistance to chemotherapeutic agents. Proc Natl Acad Sci USA 103: 11160-11165, 2006.

12. Ladelfa M, Peche LY, Toledo MF, Laiseca JE, Schneider C and Monte M: Tumor-specific MAGE proteins as regulators of p53 function. Cancer Lett 325: 11-17, 2012.

13. Doyle JM, Gao J, Wang J, Yang M and Potts PR: MAGE-RING protein complexes comprise a family of E3 ubiquitin ligases. Mol Cell 39: 963-974, 2010.

14. Gao Y, Mutter-Rottmayer E, Greenwalt AM, Goldfarb D, Yan F, Yang Y, Martinez-Chacin RC, Pearce KH, Tateishi S, Major MB and Vaziri C: A neomorphic cancer cell-specific role of MAGE-A4 in trans-lesion synthesis. Nat Commun 7: 12105, 2016.

15. Brasseur F, Rimoldi D, Liénard D, Lethé B, Carrel S, Arienti F, Suter L, Vanwijck R, Bourlond A, Humblet Y, et al: Expression of MAGE genes in primary and metastatic cutaneous melanoma. Int J Cancer 63: 375-380, 1995.

16. Bergeron A, Picard V, LaRue H, Harel F, Hovington H, Lacombe L and Fradet Y: High frequency of MAGE-A4 and MAGE-A9 expression in high-risk bladder cancer. Int J Cancer 125: 1365-1371, 2009.

17. Badovinac Črnjević T, Spagnoli G, Juretić A, Jakić-Razumović J, Podolski P and Šrić N: High expression of MAGE-A10 cancer-testis antigen in triple-negative breast cancer. Med Oncol 29: 1586-1591, 2012

18. Schultz-Thater E, Piscuoglio S, Iezzi G, Le Magnen C, Zajac P, Carafa V, Terracciano L, Tornillo L and Spagnoli GC: MAGE-A10 is a nuclear protein frequently expressed in high percentages of tumor cells in lung, skin and urothelial malignancies. Int J Cancer 129: 1137-1148, 2011.

19. Kurg R, Reinsalu O, Jagur S, Õunap K, Võsa L, Kasvandik S, Padari K, Gildemann K and Ustav M: Biochemical and proteomic characterization of retrovirus Gag based microparticles carrying melanoma antigens. Sci Rep 6: 29425, 2016.

20. Rimoldi D, Salvi S, Schultz-Thater E, Spagnoli G and Cerottini J: Anti-MAGE-3 antibody 57B and anti-MAGE-1 antibody 6C1 can be used to study different proteins of the MAGE-A family. Int J Cancer 86: 749-751, 2000.

21. Laban S, Atanackovic D, Luetkens T, Knecht R, Busch CJ, Freytag M, Spagnoli G, Ritter G, Hoffmann TK, Knuth A, et al: Simultaneous cytoplasmic and nuclear protein expression of melanoma antigen-A family and NY-ESO-1 cancer-testis antigens represents an independent marker for poor survival in head and neck cancer. Int J Cancer 135: 1142-1152, 2014.

22. Piotti KC, Scognamiglio T, Chiu R and Chen YT: Expression of cancer/testis (CT) antigens in squamous cell carcinoma of the head and neck: Evaluation as markers of squamous dysplasia. Pathol Res Pract 209: 721-726, 2013.
23. Russo AE, Torrisi E, Bevelacqua Y, Perrotta R, Libra M, McCubrey JA, Spandidos DA, Stivala F and Malaponte G: Melanoma: Molecular pathogenesis and emerging target therapies (Review). Int J Oncol 34: 1481-1489, 2009.

24. Rangwala $S$ and Tsai KY: Roles of the immune system in skin cancer. Br J Dermatol 165: 953-965, 2011.

25. Ribero S, Moscarella E, Ferrara G, Piana S, Argenziano G and Longo C: Regression in cutaneous melanoma: A comprehensive review from diagnosis to prognosis. J Eur Acad Dermatol Venereol 30: 2030-2037, 2016.

26. Bååth R: Bayesian First Aid: A package that implements Bayesian alternatives to the classical*. Test functions in R. Proc Use R, 2014.

27. Robin X, Turck N, Hainard A, Tiberti N, Lisacek F, Sanchez JC and Müller M: pROC: An open-source package for R and S+ to analyze and compare ROC curves. BMC Bioinformatics 12: 77, 2011.

28. Saito T, Wada H, Yamasaki M, Miyata H, Nishikawa $H$, Sato E, Kageyama S, Shiku H, Mori M and Doki Y: High expression of MAGE-A4 and MHC class I antigens in tumor cells and induction of MAGE-A4 immune responses are prognostic markers of CHP-MAGE-A4 cancer vaccine. Vaccine 32 : 5901-5907, 2014

29. Gjerstorff MF, Andersen MH and Ditzel HJ: Oncogenic cancer/testis antigens: Prime candidates for immunotherapy. Oncotarget 6: 15772-15787, 2015.

30. Barrow C, Browning J, MacGregor D, Davis ID, Sturrock S, Jungbluth AA and Cebon J: Tumor antigen expression in melanoma varies according to antigen and stage. Clin Cancer Res 12: 764-771, 2006.

31. Busam K, Iversen K, Berwick M, Spagnoli GC, Old LL and Jungbluth AA: Immunoreactivity with the anti-MAGE antibody 57B in malignant melanoma: Frequency of expression and correlation with prognostic parameters. Mod Pathol 13: 459-465, 2000.

32. Vinay DS, Ryan EP, Pawelec G, Talib WH, Stagg J, Elkord E, Lichtor T, Decker WK, Whelan RL, Kumara HMCS, et al: Immune evasion in cancer: Mechanistic basis and therapeutic strategies. Semin Cancer Biol 35 (Suppl): S185-S198, 2015.

33. Brisam M, Rauthe S, Hartmann S, Linz C, Brands RC, Kübler AC, Rosenwald A and Müller-Richter UD: Expression of MAGE-A1-A12 subgroups in the invasive tumor front and tumor center in oral squamous cell carcinoma. Oncol Rep 35: 1979-1986, 2016

34. Groeper C, Gambazzi F, Zajac P, Bubendorf L, Adamina M, Rosenthal R, Zerkowski HR, Heberer M and Spagnoli GC: Cancer/testis antigen expression and specific cytotoxic T lymphocyte responses in non small cell lung cancer. Int J Cancer 120: 337-343, 2007.

35. Bricard G, Bouzourene H, Martinet O, Rimoldi D, Halkic N, Gillet M, Chaubert P, Macdonald HR, Romero P, Cerottini JC and Speiser DE: Naturally acquired MAGE-A10- and SSX-2-specific CD8+ T cell responses in patients with hepatocellular carcinoma. J Immunol 174: 1709-1716, 2005.

36. Valmori D, Dutoit V, Rubio-Godoy V, Chambaz C, Liénard D, Guillaume P, Romero P, Cerottini JC and Rimoldi D: Frequent cytolytic T-cell responses to peptide MAGE-A10(254-262) in melanoma. Cancer Res 61: 509-512, 2001.

37. Lee AK and Potts PR: A comprehensive guide to the MAGE family of ubiquitin ligases. J Mol Biol 429: 1114-1142, 2017.

38. Arenberger P, Fialova A, Gkalpakiotis S, Pavlikova A, Puzanov I and Arenbergerova M: Melanoma antigens are biomarkers for ipilimumab response. J Eur Acad Dermatol Venereol 31: 252-259, 2017.

39. Zajac P, Schultz-Thater E, Tornillo L, Sadowski C, Trella E, Mengus C, Iezzi G and Spagnoli GC: MAGE-A antigens and cancer immunotherapy. Front Med (Lausanne) 4: 18, 2017.

40. Huang S, Okamoto T, Morton DL and Hoon DS: Antibody responses to melanoma/melanocyte autoantigens in melanoma patients. J Invest Dermatol 111: 662-667, 1998.

41. Fishman P, Merimski O, Baharav E and Shoenfeld Y: Autoantibodies to tyrosinase: The bridge between melanoma and vitiligo. Cancer 79: 1461-1464, 1997.

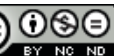

This work is licensed under a Creative Commons Attribution-NonCommercial-NoDerivatives 4.0 International (CC BY-NC-ND 4.0) License. 